\title{
Anaesthesia for Pectoralis Major Myocutaneous Flap in a Patient with a Major Facial Defect and Restricted Mouth Opening: A Case Report
}

\author{
Akwasi Antwi-Kusi, Wilfred Sam-Awortwi, Gabriel Boakye \\ Department of Anaesthesia and Intensive Care, Komfo Anokye Teaching Hospital, Kumasi, Ghana. \\ Email: antwikusi@yahoo.com \\ Received February $18^{\text {th }}, 2013$; revised March $30^{\text {th }}, 2013$; accepted April $15^{\text {th }}, 2013$ \\ Copyright (C) 2013 Akwasi Antwi-Kusi et al. This is an open access article distributed under the Creative Commons Attribution Li- \\ cense, which permits unrestricted use, distribution, and reproduction in any medium, provided the original work is properly cited.
}

\begin{abstract}
Perioperative airway management in patients with oro-facial cancers who has undergone chemo/or radiotherapy poses a great challenge to the anaesthesiologist. These challenges are mainly due to either a mass in the oral cavity or a limited mouth opening making intubation difficult or a major facial defect making mask ventilation difficult. We present our first time experience in a 27-year-old man with a malignant lymphoma of the right cheek who had undergone chemotherapy and needed plastic surgery to restore the face.
\end{abstract}

Keywords: Pectoralis Major Myocutaneous Flap; Fibreoptic Intubation; Anaesthesia; Airway

\section{Introduction}

Pectoralis major myocutaneous flap was first described by Ariyan in 1979. It is the versatile flap for the reconstruction of oral cancer surgery. It involves harvesting the pectoralis major muscle with the skin and the blood supply pulling it under the skin over the clavicle and in this case implanting it on the cheek [1,2].

The anesthesiologists have challenges in the maintenance of the airway in the perioperative period. Often the preferred route for endotracheal tube placement is tracheostomy. This may however prevent or interfere with the surgical intervention. Awaken fibreoptic nasal intubation is a suitable intervention but placement of the endotracheal tube should be off the ipsilateral chest and neck to allow the surgeon optimal operating conditions [3].

\section{Case Description}

The patient is a 37-year-old student who had a malignant lymphoma of the cheek and had undergone chemotherapy which resulted in a major defect in the right cheek and needed a facial reconstruction.

When the patient was seen in our preanaesthetic clinic, we anticipated a difficult intubation since mouth opening was very much limited. We were not keen on performing awake nasal fibreoptic intubation because of challenges we had with the only old fashioned fibreoptic broncho- scope available in our institution. Besides, since the tube had to be off the ipsilateral side of the chest and neck we only had the right nose to attempt. We therefore came to a conclusion that an elective tracheostomy should be done for maintenance of the airway.

However upon discussing our plans with the surgeon he indicated that he would rather prefer doing the tracheostomy after the operation since it will disturb his work if done before the surgery.

We therefore decided to do awake nasal fibreoptic in-

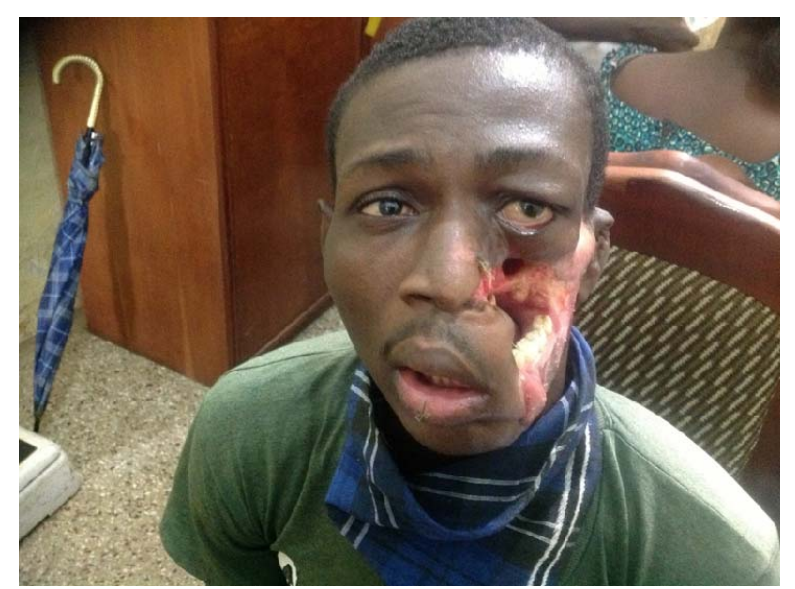

Figure 1. Patient seen at the pre-anaesthetic clinic. Note the restricred mouth opening. 
tubation. In theater we prepared our patient by setting up an IV line, $1 \mathrm{mg}$ of atropine was given intramuscularly to dry secretions in the nose. Cotton wool soaked with xylocaine with adrenaline was used to pack the nostrils to anaesthetize the mucosa and also cause vasocontiction of the vessels.

After about 40 minutes $\mathrm{SPO}_{2}$ and blood pressure were checked and the patient made to sit on the edge of the theater bed. The patient was given $100 \mathrm{mg}$ of fentanyl, a size $6.5 \mathrm{~mm}$ was passed on to the fibreoptic scope and inserted into the right nostril of the patient. On identifying the vocal cords an assistant was asked to slip the tube in and the patient was immediately given $200 \mathrm{mg}$ of propofol. The tube was carefully secured and connected to the anaesthesia machine. $6 \mathrm{mg}$ of vecuronium was given for muscle relaxation. ECG electrodes were applied at the back of the patient since the surgeon was going to work on the chest. $\mathrm{SPO}_{2}$ probe and $\mathrm{BP}$ cuff were also applied. Anaesthesia was maintained with isoflurane and oxygen/ air in the ratio or $1: 1$. Another $200 \mathrm{mcg}$ of fentanyl and $1.25 \mathrm{~g}$ novamin sulphate was given for analgesia. $200 \mathrm{mg}$ of hydrocortisone was also given.

Intraoperatively, there were no problems, the surgeon harvested the pectoralis muscle and brought it up under the skin overlying the clavicle and the neck to the right cheek. When we thought that everything was fine we realized that the chest was not moving, saturation was $100 \%$ and the BP 130/80. The ventilator was switched to the manual mode and the patient ventilated with the hand. It was at this stage detected that the endotracheal tube had been dislodged. An emergency tracheostomy was performed within five minutes and the airway was secured without any problems.

At the end of surgery, the inhalational agent was stopped. Neuromuscular relaxation reversed and the patient sent to the ICU for post operative care.

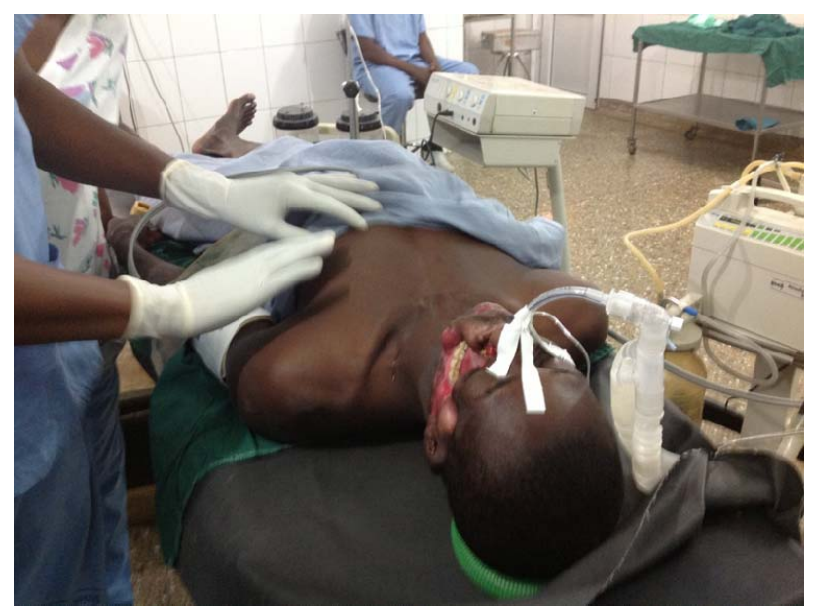

Figure 2. Patient with right naso-tracheal tube in place.

\section{Discussion}

Maxillofacial surgeries pose a great challenge to the anaesthetist [3]. Preoperative assessment of airway is the key to a successful anaesthetic management [4]. In our opinion elective tracheostomy would have been the best option for the airway management in this patient. However, there is always the need for co-operation between the anaesthesiologist and the surgeon to achieve maximum results for the patient. In this case the tracheostomy was going to interfere with the surgeon's work; he therefore requested that the patient is intubated for the surgery and tracheostomy done afterwards. Awaken intubation in compromised airway may be an excellent alternative if the procedure is carefully explained to the patient, and the anaesthetist is experienced in this procedure [5]. However if there is the need to perform awake fibreoptic intubation then it is worth securing the tube well and not to lose it in the middle of the surgery. Again preformed curved nasotracheal tube is the preferred type of tube which can be well secured and not interfere with the surgery. This type of tube was not available in our hospital. In this case what we failed to do was to suture the tube to the nares to ensure that it will stay in place throughout the surgery which lasted about 4 hours. There is also the need to prepare for tracheostomy in cases like this since normal intubation is impossible and mask ventilation likely to be difficult should the tube accidentally come out. In this case the surgeon had already prepared for tracheostomy and was able to carry it out within five minutes.

\section{Conclusion}

Management of the airway for pectoralis major mayocutaneous flap poses a unique challenge in airway management for the anaesthetist. The preferred route for endotracheal tube placement which in this case was elective tracheostomy may prevent or interfere with surgical intervention. Awaken nasal fibreoptic intubation is a suitable choice. The tube must however be sutured to the nares in order not to lose it in the course of the surgery.

\section{REFERENCES}

[1] S. Ariyan, "Further Experiences with the Pectoralis Major Myocutaneous Flap for the Immediate Repair of Defects from Excisions of Head and Neck Cancers,” Plastic \& Reconstructive Surgery, Vol. 64, 1979, pp. 605-612.

[2] S. Ariyan, "The Pectoralis Major Myocutaneous Flap. A Versatile Flap for Reconstruction in the Head and Neck," Plastic \& Reconstructive Surgery, Vol. 63, No. 1, 1979, pp. 73-81. doi:10.1097/00006534-197901000-00012

[3] M. Robert and D. William, "Comprehensive Airway Management of Patients with Maxillofacial Trauma," Craniomaxillofacial and Trauma Reconstruction, Vol. 1, No. 
1, 2008, pp. 39-47. doi:10.1055/s-0028-1098962

[4] M. S. Sarkar and V. Puri, "Faciomaxillary Surgery: Our Experience, Anaesthesiologist's Perspective,” International Journal of Anaesthesiology, Vol. 12, No. 1, 2007.

[5] American Society of Anesthesiologists Task Force on Management of the Difficult Airway, "Practice Guide- lines for Management of the Difficult Airway: An Updated Report by the American Society of Anesthesiologists Task Force on Management of the Difficult Airway,” Anesthesiology, Vol. 98, No. 5, 2003, pp. 1269-1277. doi:10.1097/00000542-200305000-00032 